\title{
NUMERICAL SOLUTIONS OF THE PROBLEM OF NON-STATIONARY PRESSURE FILTRATION WITH THE METHOD OF FINITE DIFFERENCES
} Allamuratov Sh.Z. ${ }^{1}$, Djoldasbayeva A.B. ${ }^{2}$, Allamuratov I.Sh. ${ }^{3}$ (Republic of Uzbekistan) Email: Allamuratov340@scientifictext.ru

\author{
${ }^{I}$ Allamuratov Sharapatdin Ziuatdinovich - Candidate of Physical and Mathematical Sciences, Senior Lecturer; \\ ${ }^{2}$ Djoldasbayeva Aksulu Bagitovna - Teacher, \\ DEPARTMENT OF INFORMATION EDUCATIONAL TECHNOLOGIES; \\ ${ }^{3}$ Allamuratov Ikram Sharapatdinovich - Bachelor Student, \\ NUKUS BRANCH \\ TASHKENT UNIVERSITY OF INFORMATION TECHNOLOGY NAMED AFTER MUHAMMAD AL-KHORAZMI, \\ NUKUS, REPUBLIC OF UZBEKISTAN
}

\begin{abstract}
: in this paper we consider the problem of non-stationary pressure filtration in a two-layer porous medium, when the functions are given on the boundary with time. This mathematical model was introduced a function of reducing the head, after which we moved to dimensionless variables by putting $t=\tau \cdot t_{*}, \quad x=\xi \cdot L$ and obtained the original model (1).The mathematical model is solved by the method of finite differences using the sweep method, using implicit schemes. The program is compiled on MathCad where the convergence conditions are satisfied by $\frac{h^{2}}{\tau}>0$.

Keywords: filtration, sweep method, implicit scheme, dimensionless coordinates.

\section{ЧИСЛЕННОЕ РЕШЕНИЕ ЗАДАЧИ НЕСТАЦИОНАРНОЙ НАПОРНОЙ ФИЛЬТРАЦИИ С МЕТОДОМ КОНЕЧНЫХ РАЗНОСТЕЙ Алламуратов Ш.3. ${ }^{1}$, Джолдасбаева А.Б. ${ }^{2}$, Алламуратов И.Ш. ${ }^{3}$ (Республика Узбекистан)}

\footnotetext{
${ }^{1}$ Алламуратов Шарапатдин Зиуатдинович - кандидат физико-математических наук, старший преподаватель;

${ }^{2}$ Джолдасбаева Аксулу Багитовна - преподаватель, кафедра информационных образовательных технологий; ${ }^{3}$ Алламуратов Икрам Шарапатдинович - студент бакалавриата, Нукусский филиал,

Тамкентский университет информаџионных технологий им. Мухаммада аль-Хоразми, г. Нукус, Республика Узбекистан
}

Аннотация: в данной работе рассматривается задача нестационарной напорной фильтрации в двухслойной пористой среде, когда на границе задань функции от времени. Данной математической модели введена функиия понижения напора, после чего перешли к безразмерным переменным положив $\boldsymbol{t}=\tau \cdot \boldsymbol{t}_{*}, \quad \boldsymbol{x}=\boldsymbol{\xi} \cdot \boldsymbol{L}$ и получили исходную модель (1).

Математическая модель решена методом конечных разностей с применением метода прогонки, используя неявные схемы. Программа составлена на МаthСаdе, где условия сходимости выполняются no $\frac{h^{2}}{\tau}>0$.

Ключевые слова: фильтрация, метод прогонки, неявная схема, безразмерные координаты.

В данной работе рассматривается задача фильтрации в пласте с напором $\mathrm{h}(\mathrm{x}, \mathrm{t})$ имеющая связь через слабопроницаемую прослойку с напором $\mathrm{H}$, коэфициент фильтрации которой $\mathrm{k}_{\mathrm{n}}$, и мощностью $\mathrm{m}_{\mathrm{n}}$ $[1]$.

В безразмерных переменных краевая задача имеет вид [4].

$$
\begin{gathered}
\frac{\partial s}{\partial \tau}=\frac{a t_{*}}{L^{2}} \frac{\partial^{2} s}{\partial \xi^{2}}-\frac{k_{n} a t_{*}}{m_{n} T} s, \\
s(\xi, 0)=0, \\
s(0, \tau)=\varphi_{1}\left(\tau \cdot t_{*}\right), \\
s(1, t)=\varphi_{2}\left(\tau \cdot t_{*}\right) .
\end{gathered}
$$


где безразмерные переменные

$\tau=\frac{t}{t_{*}}, \xi=\frac{x}{L}$

Выберем $\mathrm{t} *$ так, чтобы коэффициент при второй производной стал равным единице, т.е,

$t_{*}=\frac{L^{2}}{a}$.

Тогда уравнение (1) примет вид

подчиненного условиям (2)-(4).

$$
\frac{\partial s}{\partial \tau}=\frac{\partial^{2} s}{\partial \xi^{2}}-A s
$$

Здесь

$$
A=\frac{k_{n} L^{2}}{m_{n} T} .
$$

Теперь краевую задачу (1)-(4) запишем следующим образом, где нет коэффициентов перед вторыми производными

$$
\begin{gathered}
\frac{\partial s}{\partial t}=\frac{\partial^{2} s}{\partial \xi^{2}}-A s \\
s(\xi, 0)=0 \\
s(0, \tau)=B_{1}\left(1-e^{-\alpha_{1} \tau}\right) \\
s(1, \tau)=B_{2}\left(1-e^{-\alpha_{2} \tau}\right)
\end{gathered}
$$

Произведем численные расчеты с использованием метода конечных разностей. Построим разностные уравнения для первых и вторых производных входящих в исходное уравнение. Аппроксимация первой и второй частных производных производится как в [2].

$$
\frac{\partial s}{\partial \tau}=\frac{s_{i}^{j+1}-s_{i}^{j}}{\tau} ; \quad \frac{\partial^{2} s}{\partial \xi^{2}}=\frac{s_{i+1}^{j+1}-2 s_{i}^{j+1}+s_{i-1}^{j+1}}{h^{2}}
$$

Тогда (6) примет следующий вид:

$$
\frac{s_{i}^{j+1}-s_{i}^{j}}{\tau}=\frac{s_{i+1}^{j+1}-2 s_{i}^{j+1}+s_{i-1}^{j+1}}{h^{2}}-A s_{i}^{j}
$$

После несколько несложных алгебраических преобразований будем иметь следующее разностное уравнение в виде

$$
s_{i-1}^{j+1}-\left(\frac{h^{2}}{\tau}+2\right) s_{i}^{j+1}+s_{i+1}^{j+1}=-\frac{h^{2}}{\tau}(1-\tau A) s_{i}^{j},
$$

Уравнение в разностной форме (11) подчинено условиям.

$$
s_{0}^{j+1}=B_{1}\left(1-e^{-\alpha_{1} \tau}\right) ; \quad s_{N}^{j+1}=B_{2}\left(1-e^{-\alpha_{2} \tau}\right) ; \quad s_{i}^{j}=0 ; \quad i=\overline{1, N-1} .
$$

Используем общепринятые обозначения [2,3].

$a_{i}=1 ; \quad c_{i}=\frac{h^{2}}{\tau}+2 ; \quad b_{i}=1 ; \quad F_{i}=\frac{h^{2}}{\tau}(1-\tau A) s_{i}^{j}$,

В слое $j=0$ краевая задача (11)-(12) имеет следующий вид

$$
\begin{gathered}
s_{i-1}^{1}-\left(\frac{h^{2}}{\tau}+2\right) s_{i}^{1}+s_{i+1}^{1}=-\frac{h^{2}}{\tau}(1-\tau A) s_{i}^{0}, \\
s_{0}^{1}=B_{1}\left(1-e^{-\alpha_{1} \tau}\right) ; \quad s_{N}^{1}=B_{2}\left(1-e^{-\alpha_{2} \tau}\right) ; \quad s_{i}^{0}=0 ; \quad i=\overline{1, N-1} . \\
\text { где } h=\frac{1}{N} ; \quad \tau=\frac{1}{N}:
\end{gathered}
$$


Применяем прямой и обратный ход метода прогонки, используя неявную схему. Прямой ход прогонки вычисляется по следующей формуле.

$$
\begin{array}{lll}
\alpha_{i+1}=\frac{b_{i}}{c_{i}-\alpha_{i} a_{i}}, & i=1,2, \ldots N-1, & \alpha_{1}=0, \\
\beta_{i+1}=\frac{\alpha_{i} \beta_{i}+F_{i}}{c_{i}-\alpha_{i} a_{i}}, & i=1,2, \ldots N-1, & \beta_{1}=B_{1}\left(1-e^{-\alpha_{1} \tau}\right)
\end{array}
$$

Обратный ход прогонки вычисляется по следующей формуле

$$
s_{i}=\alpha_{i+1} s_{i+1}+\beta_{i+1}, \quad i=N-1, N-2, \ldots . .1 .
$$

В неявных схемах всегда выполняются условия устойчивости и сходимости по $[2,3]$

$$
\left|c_{i}\right| \geq\left|a_{i}\right|+\left|b_{i}\right|, \quad i=1,2, \ldots . . N-1 \text {. }
$$

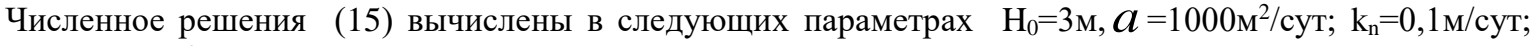
$\mathrm{m}_{\mathrm{n}}=3 \mathrm{M}, \mathrm{T}=350 \mathrm{M}^{2} /$ сут; $\mathrm{L}=200 \mathrm{M}, \mathrm{B}_{1}=0,5 \mathrm{M} ; \mathrm{B}_{2}=2,5 \mathrm{M} ; \alpha_{1}=0,3 ; \alpha_{2}=0,2$ и приведены в таблице 1 .

Таблица 1. Результаты вычисления в безразмерных координатах

\begin{tabular}{|c|c|l|l|l|c|c|c|c|c|c|c|}
\hline$\xi$ & $\tau=0$ & \multicolumn{1}{|c|}{$\tau=0,1$} & \multicolumn{1}{c|}{$\tau=0,2$} & $\tau=0,3$ & $\tau=0,4$ & $\tau=0,5$ & $\tau=0,6$ & $\tau=0,7$ & $\tau=0,8$ & $\tau=0,9$ & $\tau=1$ \\
\hline 0 & 0 & 0.02911773 & 0.05653978 & 0.08236489 & 0.10668607 & 0.12959089 & 0.151 & 0.171 & 0.191 & 0.209 & 0.226 \\
\hline 0,1 & 0 & 0.01386559 & 0.03098986 & 0.04880409 & 0.06637798 & 0.08332797 & 0.099 & 0.115 & 0.129 & 0.146 & 0.161 \\
\hline 0,2 & 0 & $8.53892469 \mathrm{e}-3$ & 0.0201237 & 0.03270769 & 0.04539784 & 0.05778199 & 0.07 & 0.081 & 0.097 & 0.113 & 0.128 \\
\hline 0,3 & 0 & $5.7533557 \mathrm{e}-3$ & 0.01388968 & 0.02291456 & 0.032117 & 0.04115191 & 0.05 & 0.067 & 0.084 & 0.1 & 0.115 \\
\hline 0,4 & 0 & $4.03398861 \mathrm{e}-3$ & $9.85504237 \mathrm{e}-3$ & 0.01638067 & 0.023073 & 0.02966427 & 0.05 & 0.069 & 0.087 & 0.104 & 0.121 \\
\hline 0,5 & 0 & $2.8837976 \mathrm{e}-3$ & $7.08800879 \mathrm{e}-3$ & 0.01182713 & 0.01670199 & 0.04097634 & 0.064 & 0.086 & 0.108 & 0.128 & 0.148 \\
\hline 0,6 & 0 & $2.08197642 \mathrm{e}-3$ & $5.13345469 \mathrm{e}-3$ & $8.58315757 \mathrm{e}-3$ & 0.03936599 & 0.06887419 & 0.097 & 0.124 & 0.15 & 0.175 & 0.199 \\
\hline 0,7 & 0 & $1.51082054 \mathrm{e}-3$ & $3.73139123 \mathrm{e}-3$ & 0.04396071 & 0.08256478 & 0.11961011 & 0.155 & 0.189 & 0.222 & 0.253 & 0.284 \\
\hline 0,8 & 0 & $1.09931144 \mathrm{e}-3$ & 0.05469051 & 0.10614958 & 0.15556192 & 0.20300948 & 0.249 & 0.292 & 0.334 & 0.375 & 0.413 \\
\hline 0,9 & 0 & 0.07222934 & 0.14161129 & 0.20825839 & 0.27227869 & 0.333776 & 0.393 & 0.45 & 0.504 & 0.556 & 0.607 \\
\hline 1 & 0 & 0.0980264 & 0.19220913 & 0.28269891 & 0.36964053 & 0.45317312 & 0.533 & 0.611 & 0.685 & 0.756 & 0.824 \\
\hline
\end{tabular}

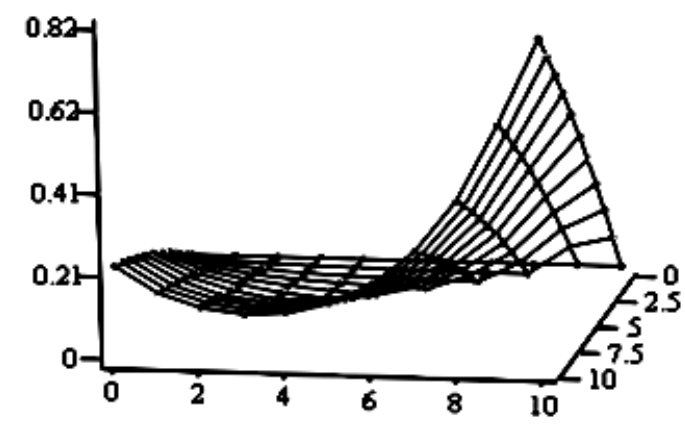

TE

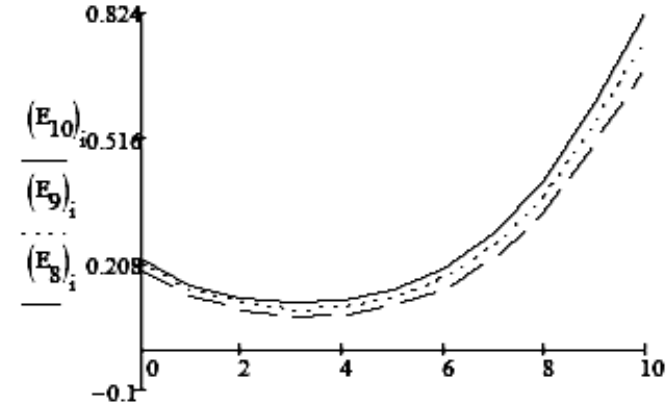

Рис. 1. Двумерный и трехмерный график в безразмерных координатах при $0 \leq \tau \leq 1,0 \leq \xi \leq 1$

\section{Список литературы / References}

1. Полубаринова-Кочина П.Я. Теория движения грунтовых вод. М.: Наука, 1977. 664 с.

2. Самарский А.А. Теория разностных схем. М.:Наука, 1977.656 с.

3. Калиткин Н.Н. Численные методы. М.: Наука, 1978. 512 с.

4. Алламуратов Ш.З., Баклушин М.Б. Использование метода конечных элементов в задаче нестационарной напорной фильтрации в слоистой пористой среде. // Узб. журн. «Проблемы информатики и энергетики». Ташкент, 2008. № 4. С. 93-98. 